Faculty of Mathematical Sciences

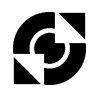

University of Twente

The Netherlands
P.O. Box 217

7500 AE Enschede

The Netherlands

Phone: +31-53-4893400

Fax: +31-53-4893114

Email: memo@math.utwente.nl

www.math.utwente.nl/publications

Memorandum No. 1622

On stability of the Hamiltonian index under contractions and closures

L. Xiong ${ }^{1}$, Z. Ryjáčé ${ }^{2}$ ANd H.J. Broersma

MARCH 2002

ISSN 0169-2690

\footnotetext{
${ }^{1}$ Dept. of Mathematics, Jiangxi Normal University, Nanchang, 330027, P.R. China

${ }^{2}$ Dept. of Mathematics, University of West Bohemia, and Institute of Theoretical Computer Science (ITI), Charles University, P.O. Box 314, 30614 Pilsen, Czech Republic
} 


\title{
On stability of the hamiltonian index under contractions and closures
}

\author{
Liming Xiong ${ }^{1,2}$ \\ Zdeněk Ryjáček ${ }^{3,4}$ \\ Hajo Broersma ${ }^{5}$
}

January 14,2002

\begin{abstract}
The hamiltonian index of a graph $G$ is the smallest integer $k$ such that the $k$-th iterated line graph of $G$ is hamiltonian. We first show that, with one exceptional case, adding an edge to a graph cannot increase its hamiltonian index. We use this result to prove that neither the contraction of an $A_{G}(F)$-contractible subgraph $F$ of a graph $G$ nor the closure operation performed on $G$ (if $G$ is claw-free) affects the value of the hamiltonian index of a graph $G$.
\end{abstract}

Keywords: hamiltonian index, stable property, closure of a graph, contractible graph, collapsible graph

AMS Subject Classification (2000): 05C45, 05C35

\section{Introduction}

In this paper, we consider only finite undirected loopless graphs $G=(V(G), E(G))$. However, except for Section 4, we admit $G$ to have multiple edges. We generally follow the most common graph-theoretical notation and terminology and for concepts and notations not defined here we refer the reader to [1].

A dominating closed trail (abbreviated DCT) in a graph $G$ is a closed trail (or, equivalently, an eulerian subgraph) $T$ in $G$ such that every edge of $G$ has at least one vertex on $T$. The following result by Harary and Nash-Williams relates the existence of a DCT in a graph $G$ and the existence of a hamiltonian cycle in its line graph $L(G)$. Here the

\footnotetext{
${ }^{1}$ Department of Mathematics, Jiangxi Normal University, Nanchang, 330027, P. R. China, e-mail liming_xiong@hotmail.com

${ }^{2}$ Research partially supported by Natural Science Fund of Jiangxi Provice and by grant No. LN00A056 of the Czech Ministry of Education.

${ }^{3}$ Department of Mathematics, University of West Bohemia, and Institute of Theoretical Computer Science (ITI), Charles University, P.O. Box 314, 30614 Pilsen, Czech Republic, e-mail ryjacek@kma.zcu.cz

${ }^{4}$ Research supported by grant No. LN00A056 of the Czech Ministry of Education.

${ }^{5}$ Faculty of Mathematical Sciences, University of Twente, P.O. Box 217, 7500 AE Enschede, The Netherlands, e-mail broersma@math.utwente.nl
} 
line graph of a graph $G$, denoted by $L(G)$, is the graph with vertex set $E(G)$ and with two vertices adjacent in $L(G)$ if and only if the corresponding edges of $G$ have a vertex in common.

Theorem A [5]. Let $G$ be a graph with at least three edges. Then $L(G)$ is hamiltonian if and only if $G$ has a $D C T$.

If $P=x_{1}, \ldots, x_{k}$ is a path in a graph $G$ and $S, T \subset G$ are subgraphs of $G$, then we say that $P$ is an $(S, T)$-path if $x_{1} \in V(S)$ and $x_{k} \in V(T)$. The distance of two subgraphs $S, T \subset G$ (denoted $\left.\operatorname{dist}_{G}(S, T)\right)$ is the minimum length of an $(S, T)$-path. For any integer $i \geq 0$ set $V_{i}(G)=\left\{v \in V(G): d_{G}(v)=i\right\}$ (here $d_{G}(v)$ denotes the degree of a vertex $v$ in $G$ ) and $W(G)=V(G) \backslash V_{2}(G)$. A branch in $G$ is a nontrivial path with endvertices in $W(G)$ and with internal vertices, if any, of degree 2 in $G$ (and thus not in $W(G)$ ). If a branch has length 1 , then it has no internal vertex. Let $B(G)$ denote the set of branches of $G$, and let $B_{1}(G)$ be the subset of $B(G)$ in which every branch has an end in $V_{1}(G)$. For any subgraph $H$ of $G$ let $B_{H}(G)$ be the set of those branches of $G$ which have all edges in $H$.

If $G$ is a graph and $k \geq 2$ an integer, then $E U_{k}(G)$ denotes the set of all subgraphs $H$ of $G$ that satisfy the following conditions:

(I) $d_{H}(x) \equiv 0(\bmod 2)$ for every $x \in V(H)$;

(II) $V_{0}(H) \subseteq \bigcup_{i-3}^{\Delta(G)} V_{i}(G) \subseteq V(H)$;

(III) $\operatorname{dist}_{G}\left(H_{1}, \stackrel{i=3}{H}-H_{1}\right) \leq k-1$ for every subgraph $H_{1}$ of $H$;

(IV) $|E(b)| \leq k+1$ for every branch $b \in B(G) \backslash B_{H}(G)$;

(V) $|E(b)| \leq k$ for every branch $b \in B_{1}(G)$.

The following theorem, which can be considered as an analogue of Theorem A for the $k$-th iterated line graph $L^{k}(G)$ of a graph $G$, shows the importance of subgraphs from $E U_{k}(G)$. Here $L^{k}(G)$ is defined recursively by $L^{0}(G)=G, L^{1}(G)=L(G)$ and $L^{k}(G)=L\left(L^{k-1}(G)\right)$.

Theorem B [13]. Let $G$ be a connected graph with at least three edges and let $k \geq 2$ be an integer. Then $L^{k}(G)$ is hamiltonian if and only if $E U_{k}(G) \neq \emptyset$.

The hamiltonian index of a graph $G$, denoted $h(G)$, is the smallest integer $k$ such that the $k$-th iterated line graph $L^{k}(G)$ of $G$ is hamiltonian. Thus, Theorem B equivalently says that for an integer $k \geq 2$ and for any graph $G, h(G) \leq k$ if and only if $E U_{k}(G) \neq \emptyset$.

If $F$ is a subgraph of a graph $G$, then a vertex $x$ is said to be a vertex of attachment of $F$ in $G$ if $x \in V(F)$ and $x$ has a neighbor in $V(G) \backslash V(F)$. The set of all vertices of attachment of a subgraph $F$ in $G$ is denoted by $A_{G}(F)$.

For a subgraph $F \subset G$ of a graph $G,\left.G\right|_{F}$ denotes the graph obtained from $G$ by identifying the vertices of $F$ as a (new) vertex $v_{F}$, and by replacing the created loops by pendant edges (i.e. edges with one vertex of degree 1) attached to $v_{F}$. We say that the graph $\left.G\right|_{F}$ was obtained from $G$ by contracting the subgraph $F$ (observe that $\left.|E(G)|=\left|E\left(\left.G\right|_{F}\right)\right|\right)$.

If $G$ is a graph, $X \subset V(G)$ and $\mathcal{A}$ is a partition of $X$ into subsets, then $E(\mathcal{A})$ denotes the set of all edges $a_{1} a_{2}$ (not necessarily in $E(G)$ ) such that $a_{1}, a_{2}$ are in the same 
element of $\mathcal{A}$, and $G^{\mathcal{A}}$ denotes the graph with vertex set $V\left(G^{\mathcal{A}}\right)=V(G)$ and edge set $E\left(G^{\mathcal{A}}\right)=E(G) \cup E(\mathcal{A})$. Note that $E(G)$ and $E(\mathcal{A})$ are considered to be disjoint, i.e., if $e_{1}=a_{1} a_{2} \in E(G)$ and $e_{2}=a_{1} a_{2} \in E(\mathcal{A})$, then $e_{1}, e_{2}$ are parallel edges in $G^{\mathcal{A}}$.

Let $F$ be a graph and let $A \subset V(F)$. Following [11], we say that the graph $F$ is $A$-contractible, if for every even subset $X \subset A$ and for every partition $\mathcal{A}$ of $X$ into twoelement subsets the graph $F^{\mathcal{A}}$ has a DCT containing all vertices of $A$ and all edges of $E(\mathcal{A})$. Note that this definition allows $X$ to be empty, in which case $F^{\mathcal{A}}=F$. Also, if $F$ is $A$-contractible, then $F$ is $A^{\prime}$-contractible for any $A^{\prime} \subset A$ (since every subset $X$ of $A^{\prime}$ is a subset of $A$ ).

Set $d_{T}(G)=\max \{|S|: S \subset E(G)$ and there is a closed trail $T \subset G$ such that every edge $e \in S$ has at least one vertex on $T\}$. The following result was proved in [11].

Theorem C [11]. Let $F$ be a connected graph and let $A \subset V(F)$. Then $F$ is $A$ contractible if and only if

$$
d_{T}(G)=d_{T}\left(\left.G\right|_{F}\right)
$$

for every graph $G$ such that $F \subset G$ and $A_{G}(F)=A$.

For $d_{T}(G)=|E(G)|$ we get the following immediate corollary.

Corollary D [11]. Let $G$ be a graph and let $F \subset G$ be an $A_{G}(F)$-contractible subgraph of $G$. Then $G$ has a DCT if and only if $\left.G\right|_{F}$ has a DCT.

Note that $\left.G\right|_{F}$ may contain multiple edges even if $G$ is a simple graph. However, it is easy to observe that a multiple edge is a contractible subgraph and hence, by a series of subsequent contractions, it is always possible to reduce $\left.G\right|_{F}$ to a certain simple graph $G^{\prime}$ with $d_{T}\left(G^{\prime}\right)=d_{T}\left(\left.G\right|_{F}\right)=d_{T}(G)$.

We say that a graph $G$ is claw-free if $G$ is a simple graph that does not contain a copy of the claw as an induced subgraph. It is well-known that every line graph is claw-free.

Let $G$ be a claw-free graph. A vertex $x \in V(G)$ is locally connected if $G[N(x)]$ is a connected graph. For $x \in V(G)$, the graph $G_{x}^{\prime}$ with vertex set $V\left(G_{x}^{\prime}\right)=V(G)$ and edge set $E\left(G_{x}^{\prime}\right)=E(G) \cup\{x y \mid x, y \in N(x)\}$ is called the local completion of $G$ at $x$. It was shown in [9] that the local completion of a claw-free graph $G$ at $x$ is again claw-free, and if $x$ is a locally connected vertex, then $c\left(G_{x}^{\prime}\right)=c(G)$ (where $c(G)$ denotes the circumference of $G$, i.e. the length of a longest cycle in $G$ ).

The following concept was introduced in [9]. Let $G$ be a claw-free graph and let $c l(G)$ be a graph obtained from $G$ by recursively performing the local completion operation at locally connected vertices with noncomplete neighborhood, as long as this is possible. The graph $\mathrm{cl}(G)$ is called the closure of the graph $G$. The following theorem summarizes basic properties of the closure operation.

Theorem E [9]. Let $G$ be a claw-free graph. Then

(i) $\operatorname{cl}(G)$ is uniquely determined,

(ii) $c(c l(G))=c(G)$,

(iii) $\operatorname{cl}(G)$ is the line graph of a triangle-free graph. 
Theorem $\mathrm{E}$ has the following immediate consequence.

Corollary F [9]. Let $G$ be a claw-free graph. Then $G$ is hamiltonian if and only if $\operatorname{cl}(G)$ is hamiltonian.

If $\mathcal{C}$ is a class of graphs, $\Gamma$ is a graph operation on $\mathcal{C}$ and $\mathcal{P}$ is a graph property, then $\mathcal{P}$ is said to be stable under $\Gamma$ if, for any $G \in \mathcal{C}, G$ has $\mathcal{P}$ if and only if $\Gamma(G)$ has $\mathcal{P}$. Similarly, a graph invariant $\pi$ is said to be stable under $\Gamma$ if for any $G \in \mathcal{C}$ we have $\pi(G)=\pi(\Gamma(G))$. In this terminology, Theorem $\mathrm{C}$ and Corollary D say that $d_{T}(G)$ and the existence of a DCT are stable under the operation of contraction of an $A_{G}(F)$-contractible subgraph $F$, and Theorem $\mathrm{E}$ and Corollary $\mathrm{F}$ say that the circumference and hamiltonicity are stable under the closure operation on claw-free graphs. Stability of some further graph properties and invariants under the closure operation was studied e.g. in [2], [10], [6] or [8] (see also the survey paper [3]).

The main results of this paper, Theorems 7 and 10 , show that the hamiltonian index is stable under the operation of contraction of an $A_{G}(F)$-contractible subgraph $F$ and under the closure operation on claw-free graphs.

\section{The hamiltonian index of a subgraph}

Our first result shows that, with one exceptional case, adding an edge to a graph cannot increase its hamiltonian index.

Theorem 1. Let $G$ be a connected graph with three edges other than a path. Then for any two vertices $a, b$ of $V(G)$ with $d_{G}(a)+d_{G}(b) \geq 3$, either $h(G)=1$ and $h(G+a b)=2$ or $h(G) \geq h(G+a b)$. Moreover, if $\operatorname{dist}_{G}(a, b)=2$, then

$$
h(G) \geq h(G+a b) .
$$

Proof. Let $G^{\prime}=G+a b$. We distinguish the following cases.

Case 1: $h\left(G^{\prime}\right)=0$. Then $h(G) \geq 0=h\left(G^{\prime}\right)$.

Case 2: $h\left(G^{\prime}\right)=1$. Then $G^{\prime}$ is not hamiltonian but $L\left(G^{\prime}\right)$ is hamiltonian, implying that $G$ is also not hamiltonian. Hence $h(G) \geq 1=h\left(G^{\prime}\right)$.

Case 3: $h\left(G^{\prime}\right) \geq 2$.

If $h(G)=0$, then $G$ is hamiltonian and since $V(G)=V\left(G^{\prime}\right)$, we have $h\left(G^{\prime}\right)=0$, a contradiction.

Suppose next $h(G)=1$. Then, by Theorem A, $G$ has a DCT $T$. Since $h\left(G^{\prime}\right) \geq 2, T$ is not a DCT of $G^{\prime}$. Hence both $a$ and $b$ are not in $V(T)$, and necessarily both $a$ and $b$ have a neighbor on $T$. This implies that any hamiltonian cycle in $L(G)$ is a DCT in $L\left(G^{\prime}\right)$, implying that $h\left(G^{\prime}\right) \leq 2$. Since, by the assumption, $h\left(G^{\prime}\right) \geq 2$, we have $h(G)=1$ and $h\left(G^{\prime}\right)=2$.

Now, for $a, b \in V(G)$ with $\operatorname{dist}_{G}(a, b)=2$, both $a$ and $b$ are not in $V(T)$ and hence there is a vertex $c_{a b}$ in $N_{G}(a) \cap N_{G}(b)$ with $c_{a b} \in V(T)$. Let $T^{\prime}$ be a closed trail in $G^{\prime}$ 
obtained from $T$ by adding the cycle $c_{a b} a b c_{a b}$. Then $T^{\prime}$ is a DCT in $G^{\prime}$, implying $h\left(G^{\prime}\right) \leq 1$, a contradiction.

Hence we can suppose that $h(G) \geq 2$ and $d_{G}(a)+d_{G}(b) \geq 3$. By Theorem B, there is a subgraph $H \in E U_{h(G)}(G)$. Let $H^{\prime}$ be the subgraph of $G^{\prime}$ with vertex set

$$
V\left(H^{\prime}\right)=V(H) \cup\left\{v \in\{a, b\}: d_{G^{\prime}}(v) \geq 3\right\}
$$

and edge set

$$
E\left(H^{\prime}\right)=E(H) .
$$

We will show that $H^{\prime} \in E U_{h(G)}\left(G^{\prime}\right)$, i.e., $H^{\prime}$ satisfies the conditions (I) - (V) of the definition of $E U_{h(G)}\left(G^{\prime}\right)$ (for the graph $G^{\prime}$ and $k=h(G)$ ). Obviously, $H^{\prime}$ satisfies conditions (I) and (II).

If one of $a, b$ has degree 1 in $G$, say, $d_{G}(a)=1$, then $d_{G}(b) \geq 2$ since $d_{G}(a)+d_{G}(b) \geq 3$. The branch of $B_{1}(G)$, containing $a$ (denoted by $P$ ) will become a new branch $P^{\prime}=P b$ in $B\left(G^{\prime}\right) \backslash\left(B_{H^{\prime}}\left(G^{\prime}\right) \cup B_{1}\left(G^{\prime}\right)\right)$ of length $|E(P)|+1 \leq h(G)+1$. The other branches of $B\left(G^{\prime}\right) \backslash B_{H^{\prime}}(G)$ are the same as those of $B(G) \backslash B_{H}(G)$ except the only case that $d_{G}(b)=2$ and $b$ is not in $V(H)$; in this exceptional case, the branch containing $b$ turns into two shorter branches in $B\left(G^{\prime}\right) \backslash B_{H^{\prime}}\left(G^{\prime}\right)$. This shows that $H^{\prime}$ satisfies (IV) and (V). If both $a$ and $b$ have degree at least 2 in $G$, then the branches in $B\left(G^{\prime}\right) \backslash B_{H^{\prime}}\left(G^{\prime}\right)$ are the same as those in $B(G) \backslash B_{H}(G)$ except the case that $a$ or $b$ (or both) have degree exactly 2 in $G$ and they are not in $V(H)$; in this exceptional case, the branches in $B\left(G^{\prime}\right) \backslash B_{H^{\prime}}\left(G^{\prime}\right)$ will be shorter than those in $B(G) \backslash B_{H}(G)$. This shows that $H^{\prime}$ satisfies (IV) and $(\mathrm{V})$.

It remains to show that $H^{\prime}$ satisfies (III). Suppose there is a subgraph $H_{1}^{\prime}$ of $H^{\prime}$ such that $\operatorname{dist}_{G^{\prime}}\left(H_{1}^{\prime}, H^{\prime}-H_{1}^{\prime}\right) \geq h(G) \geq 2$. It is easy to see that $V\left(H_{1}^{\prime}\right) \cap V(H)$ and $V\left(H^{\prime}-\right.$ $\left.H_{1}^{\prime}\right) \cap V(H)$ cannot be both empty. Suppose first that $V\left(H_{1}^{\prime}\right) \cap V(H)=\emptyset$ and $V\left(H^{\prime}-\right.$ $\left.H_{1}^{\prime}\right) \cap V(H) \neq \emptyset$ (note that the case that $V\left(H_{1}^{\prime}\right) \cap V(H) \neq \emptyset$ and $V\left(H^{\prime}-H_{1}^{\prime}\right) \cap V(H)=\emptyset$ is symmetric). Then $V\left(H_{1}^{\prime}\right) \subseteq\{a, b\}$. If $V\left(H_{1}^{\prime}\right)=\{a, b\}$, then $d_{G}(a), d_{G}(b) \leq 2$ since $\{a, b\} \cap V(H)=\emptyset$ and $H$ satisfies (II). By the definition of $H^{\prime}, d_{G^{\prime}}(a), d_{G^{\prime}}(b) \geq 3$. Hence $d_{G}(a)=d_{G}(b)=2$, implying that both $a$ and $b$ are on some branches of $B(G) \backslash B_{H}(G)$. Since $H$ satisfies (IV) and (V), $\operatorname{dist}_{G}(\{a, b\}, H) \leq h(G)-1$; in this case, any shortest $(\{a, b\}, H)$-path in $G$ is also an $\left(H_{1}^{\prime}, H^{\prime}-H_{1}^{\prime}\right)$-path in $G^{\prime}$. Hence $\operatorname{dist}_{G^{\prime}}\left(H_{1}^{\prime}, H^{\prime}-H_{1}^{\prime}\right) \leq$ $\operatorname{dist}_{G}(\{a, b\}, H) \leq h(G)-1$, a contradiction. This implies that $H_{1}^{\prime}$ has exactly one vertex, say, $V\left(H_{1}^{\prime}\right)=\{a\}$. Similarly, $\operatorname{dist}_{G}(\{a\}, H) \leq h(G)-1$ and any shortest $(\{a\}, H)$-path in $G$ is an $\left(H_{1}^{\prime}, H^{\prime}-H_{1}^{\prime}\right)$-path in $G^{\prime}$, implying that $\operatorname{dist}_{G^{\prime}}\left(H_{1}^{\prime}, H^{\prime}-H_{1}^{\prime}\right) \leq \operatorname{dist}_{G}(\{a\}, H) \leq$ $h(G)-1$, a contradiction. Finally, suppose that both $V\left(H_{1}^{\prime}\right) \cap V(H)$ and $V\left(H^{\prime}-H_{1}^{\prime}\right) \cap V(H)$ are nonempty, and set $H_{1}=H_{1}^{\prime} \cap H$. In this case, any shortest $\left(H_{1}, H-H_{1}\right)$-path in $G$ is also an $\left(H_{1}^{\prime}, H^{\prime}-H_{1}^{\prime}\right)$-path in $G^{\prime}$. Hence $\operatorname{dist}_{G^{\prime}}\left(H_{1}^{\prime}, H^{\prime}-H_{1}^{\prime}\right) \leq \operatorname{dist}_{G}\left(H_{1}, H-H_{1}\right) \leq$ $h(G)-1$, a contradiction. This shows that $H^{\prime}$ satisfies (III). Thus $H^{\prime} \in E U_{h(G)}\left(G^{\prime}\right)$, implying $h\left(G^{\prime}\right) \leq h(G)$.

If $\operatorname{dist}_{G}(a, b)=2$ and $d_{G}(a)+d_{G}(b)=2$, then both $a$ and $b$ are on branches of length 1 which are all in $B_{1}(G)$. Repeating the above argument, we can prove that $h(G) \geq h(G+a b)$ for $a$ and $b$ with $\operatorname{dist}_{G}(a, b)=2$.

Example 2. We construct an infinite family of graphs showing that the assumption $d_{G}(a)+d_{G}(b) \geq 3$ in Theorem 1 cannot be relaxed. Let $C$ be a cycle of length $|E(C)| \geq 6$ 
and let $x, y$ be two vertices on $C$ with maximum $\operatorname{dist}_{C}(x, y)$. Take two disjoint paths $P_{1}, P_{2}$ with endvertices $x^{\prime}, a$ and $y^{\prime}, b$, respectively. Let $G$ be the graph obtained from $C$ and $P_{1}, P_{2}$ by identifying $x^{\prime}, x$ and $y^{\prime}, y$ respectively (for $|E(C)|=6$ see Figure $1($ a $)$ ). It is easy to see that $P_{1}$ and $P_{2}$ are two branches in $B_{1}(G)$. If $\left|E\left(P_{1}\right)\right|,\left|E\left(P_{2}\right)\right| \leq(|E(C)|-2) / 4$, then $h(G)=\max \left\{\left|E\left(P_{1}\right)\right|,\left|E\left(P_{2}\right)\right|\right\}$ (see [12] and [13]) and $h(G+a b)=\left|E\left(P_{1}\right)\right|+\left|E\left(P_{2}\right)\right|=$ $h(G)+\min \left\{\left|E\left(P_{1}\right)\right|,\left|E\left(P_{2}\right)\right|\right\}>h(G)$ (see [12] and [14]).

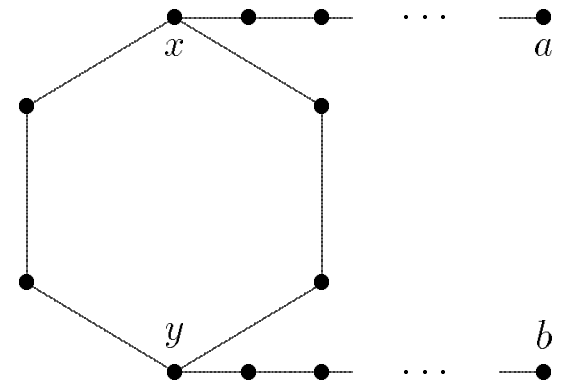

(a)

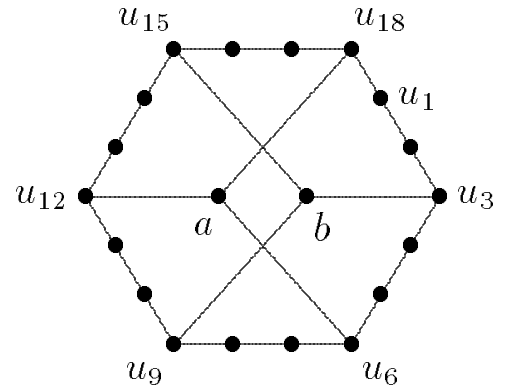

(b)

Figure 1

Remark 3. In fact, using the method of proof of Theorem 1 (with just a slight modification of the proof of (IV) and (V)), it would be possible to show that without the assumption $d_{G}(a)+d_{G}(b) \geq 3$ one can still prove that $2 h(G) \geq h(G+a b)$. The graph $G$ from Example 2 with $\left|E\left(P_{1}\right)\right|=\left|E\left(P_{2}\right)\right| \leq(|E(C)|-2) / 4$ gives $2 h(G)=h(G+a b)$ (see [14]), which shows that this inequality is sharp.

Using a similar modification of the proof of Theorem 1, it would be also possible to prove that $2 h(G) \geq h\left(G^{\prime}\right)$ if $G$ is a spanning subgraph of $G^{\prime}$. Details are left to the reader.

Example 4. Without the condition $\operatorname{dist}_{G}(a, b)=2$, we can construct a graph $G$ such that $h(G)=1$ and $h(G+a b)=2$ even under the condition that $d_{G}(a)+d_{G}(b) \geq 3$. Let $t, s \geq 3$ be integers, let $C=u_{1} u_{2} \cdots u_{t} \cdots u_{2 t} \cdots u_{s t} u_{1}$ be a cycle of length st and let $a$ and $b$ be two distinct vertices that are not on $C$. The graph $G$ is obtained from $C$ and $a, b$ by adding $s$ new edges between $a, b$ and $u_{t}, u_{2 t}, \cdots u_{s t}$ such that each of $a, b$ is adjacent to at least one and each of $u_{t}, u_{2 t}, \cdots, u_{s t}$ is adjacent to exactly one of the new edges (for $t=3, s=6$ and one of the possible choices of the new edges see Figure 1(b)). By the construction, $d_{G}(a)+d_{G}(b)=s \geq 3$. It is easy to see (by Theorem A) that $h(G)=1$ and $h(G+a b)=2$.

The following corollary is easily obtained from Theorem 1.

Corollary 5. Let $G$ be a connected graph with at least three edges other than a path and $G^{\prime}$ be a graph obtained from $G$ by recursively adding the edges whose ends $a$ and $b$ satisfy the assumptions of the first part of Theorem 1. Then either $h(G)=1$ and $h\left(G^{\prime}\right)=2$, or $h(G) \geq h\left(G^{\prime}\right)$. 


\section{The hamiltonian index is stable under contraction}

We begin this section with the following easy observation which will be used in our proof.

Lemma 6. Let $G$ be a graph with $h(G) \geq 2$. For any $H \in E U_{h(G)}(G)$ and any subgraph $H_{1}$ of $H$, if the distance between $H_{1}$ and $H-H_{1}$ is at least 2, then the shortest path of $G$ between $H_{1}$ and $H-H_{1}$ is a branch of $G$, whose ends are not adjacent in $G$.

Proof. The lemma follows easily from the condition (II) of the definition of $E U_{h(G)}(G)$.

We will also need the following well-known result.

Theorem G [7]. A connected graph is eulerian if and only if each minimum edge cut contains an even number of edges.

If $G$ is a hamiltonian graph (i.e. $h(G)=0$ ) and $F \subset G$ is a nontrivial subgraph of $G$, then $\left.G\right|_{F}$ cannot be hamiltonian (since it has connectivity 1 ), and it is easy to observe that any hamiltonian cycle in $G$ turns into a DCT in $\left.G\right|_{F}$. Hence $h(G)=0$ implies $h\left(\left.G\right|_{F}\right)=1$ for any nontrivial subgraph $F \subset G$. However, the following theorem shows that for $h(G) \geq 1$, i.e. for nonhamiltonian graphs, the hamiltonian index is stable under contraction of a contractible subgraph.

Theorem 7. Let $G$ be a nonhamiltonian graph other than a path and $F$ be an $A_{G}(F)$ contractible subgraph of $G$. Then $h(G)=h\left(\left.G\right|_{F}\right)$.

Proof. Let $G^{\prime}=\left.G\right|_{F}$. By Theorems A and C, $h(G) \leq 1$ if and only if $h\left(G^{\prime}\right) \leq 1$. Equivalently, $h(G) \geq 2$ if and only if $h\left(G^{\prime}\right) \geq 2$. It is sufficient to consider the case that $h(G) \geq 2$. Hence $h\left(G^{\prime}\right) \geq 2$. We first prove that $h\left(G^{\prime}\right) \leq h(G)$. By Theorem B and $h(G) \geq 2$, we can take a subgraph $H$ in $E U_{h(G)}(G)$. Let $H^{\prime}$ be the graph obtained from $\left.H\right|_{F}$ by deleting the new pendant edges. We prove that $H^{\prime}$ is in $E U_{h(G)}\left(G^{\prime}\right)$, i.e., $H^{\prime}$ satisfies the conditions of the definition of $E U_{h(G)}\left(G^{\prime}\right)$ for the graph $G^{\prime}$ and $k=h(G)$. By Theorem G, $H^{\prime}$ satisfies (I) and (II) in the definition of $E U_{h(G)}\left(G^{\prime}\right)$.

The following claim is immediate from the definitions of $A_{G}(F)$ and $A$-contractible graph.

Claim 1. Every vertex in $A_{G}(F)$ has degree at least 3 in $G$.

Now Claim 1 and Lemma 6 easily imply that $H^{\prime}$ satisfies also the other conditions in the definition of $E U_{G}\left(G^{\prime}\right)$, and hence $h\left(G^{\prime}\right) \leq h(G)$.

We prove that $h(G) \leq h\left(G^{\prime}\right)$. Since $h\left(G^{\prime}\right) \geq 2$, by Theorem B, we can take a subgraph $H^{\prime}$ in $E U_{h\left(G^{\prime}\right)}\left(G^{\prime}\right)$. Set $V_{b}\left(H^{\prime}\right)=\left\{x \in F: x\right.$ is an endvertex of a branch of $\left.B_{H^{\prime}}(G)\right\}$. Let $r(x)$ denote the number of branches of $B_{H^{\prime}}(G)$, one of which has $x$ as an endvertex. Set $V_{b}^{j}=\left\{x \in V_{b}\left(H^{\prime}\right): r(x) \equiv j(\bmod 2)\right\}$. Since $H^{\prime}$ satisfies $(\mathrm{I}), \sum_{x \in V_{b}^{1}} r(x)+\sum_{x \in V_{b}^{2}} r(x)=$ $\sum_{x \in V_{b}} r(x)=d_{H^{\prime}}\left(v_{F}\right)$ is even. Since $\sum_{x \in V_{b}^{2}} r(x)$ is even, it follows that $\sum_{x \in V_{b}^{1}} r(x)$ is also even. Hence $\left|V_{b}^{1}\right|$ is even. Let $X=V_{b}^{1}$. Take one partition $\mathcal{A}$ of $X$ into two-element 
subsets. Since $F$ is $A_{G}(F)$-contractible, $F^{\mathcal{A}}$ has a DCT $T$ containing all vertices of $A_{G}(F)$ and all edges of $E(\mathcal{A})$. Now we let $H$ be the graph with vertex set

$$
V(H)=V\left(H^{\prime}\right) \cup\left(\bigcup_{i=3}^{\Delta(G)} V_{i}(G)\right) \cup V(T)
$$

and edge set

$$
E(H)=E\left(H^{\prime}\right) \cup(E(T) \backslash E(\mathcal{A})) .
$$

We prove that $H \in E U_{h\left(G^{\prime}\right)}(G)$. Obviously, $H$ satisfies the conditions (I) and (II) in the definition of $E U_{h\left(G^{\prime}\right)}(G)$. Since $T$ is a DCT which contains all vertices of $A_{G}(F)$ and all edges of $E(\mathcal{A})$, by Claim $1, H$ satisfies (IV) and (V). By Lemma $6, H$ satisfies (III). Hence $H \in E U_{h\left(G^{\prime}\right)}(G)$, implying $h(G) \leq h\left(G^{\prime}\right)$. This completes the proof of Theorem 7 .

Remark 8. Catlin [4] introduced a reduction technique based on the concept of a collapsible graph. It was shown in [11] that every collapsible graph $F$ is $V(F)$-contractible. Thus, Theorem 7 implies that the hamiltonian index is stable under contraction of a collapsible subgraph.

\section{The hamiltonian index of a claw-free graph is sta- ble under the closure}

In this section we assume all graphs to be simple (i.e. without multiple edges).

Lemma 9. Let $G$ be a connected claw-free graph with at least three edges other than a path. Then

(i) $h(G)=0$ if and only if $h(\operatorname{cl}(G))=0$;

(ii) $h(G)=1$ if and only if $h(\operatorname{cl}(G))=1$.

Proof. By Corollary F, it is sufficient to prove that $h(G) \leq 1$ if and only if $h(\operatorname{cl}(G)) \leq 1$. Since $V(\operatorname{cl}(G))=V(G)$, using Theorem 1 we obtain $h(\operatorname{cl}(G)) \leq h(G)$. Hence $h(G) \leq 1$ implies $h(\operatorname{cl}(G)) \leq 1$.

Conversely, suppose that $h(\operatorname{cl}(G)) \leq 1$, i.e., by Theorem $\mathrm{A}, \operatorname{cl}(G)$ has a DCT. We prove that $G$ also has a DCT. It is sufficient to prove that if there is a DCT in $G^{\prime}=G+x y$ for any pair of vertices $x$ and $y$ with $x y \notin E(G)$ such that they have a common neighbor $c_{x y}$ in $G$ which is a locally connected vertex of $G$, then there is also a DCT in $G$. Let $P$ be a shortest $(x, y)$-path in $G\left[N_{G}\left(c_{x y}\right)\right]$. Since $G$ is claw-free and $P$ is chordless, $|E(P)| \leq 3$. Since $x y \notin E(G), 2 \leq|E(P)| \leq 3$. Let $F=G\left[V(P) \cup\left\{c_{x y}\right\}\right]$ and $F^{\prime}=G^{\prime}\left[V(P) \cup\left\{c_{x y}\right\}\right]$. Then $F$ is isomorphic to the graph $F_{1}$ or $F_{2}$ and $F^{\prime}$ is isomorphic to the graph $F_{3}$ or $F_{4}$ of Figure 2.

It is easy to verify that each of the graphs $F_{i}$ is $V\left(F_{i}\right)$-contractible, $i=1,2,3,4$. Let $e$ be one of the (at least 6 if $F^{\prime} \simeq F_{3}$ or at least 8 if $F^{\prime} \simeq F_{4}$ ) pendant edges of $G^{\prime}$ adjacent to the vertex $v_{F}$. Since $\left.\left.G\right|_{F} \simeq G^{\prime}\right|_{F^{\prime}}-e$ and clearly $\left.G^{\prime}\right|_{F^{\prime}}$ has a DCT if and only if $\left.G^{\prime}\right|_{F^{\prime}}-e$ has a DCT, by Corollary D, $G^{\prime}$ has a DCT if and only if $G$ has a DCT. Hence the lemma follows. 

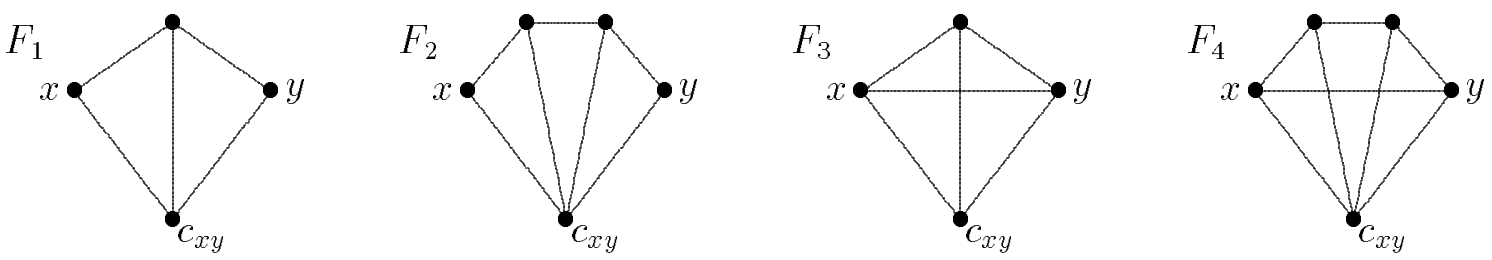

Figure 2

The following result, which is the main result of this section, shows that the hamiltonian index is stable under the closure operation in claw-free graphs.

Theorem 10. Let $G$ be a connected claw-free graph with at least three edges other than a path. Then

$$
h(G)=h(\operatorname{cl}(G))
$$

Proof. By Lemma 9, we only need to prove the case that $h(G) \geq 2$. Since $G \subseteq$ $\operatorname{cl}(G)$ and $V(G)=V(\operatorname{cl}(G))$, we have $h(G) \geq h(\operatorname{cl}(G))$ by the definition of $\operatorname{cl}(G)$ and by Theorem 1. It remains to prove that $h(G) \leq h(\mathrm{cl}(G))$. It is sufficient to prove that $h(G) \leq h(G+x y)$ for any pair of vertices $x$ and $y$ with $x y \notin E(G)$ such that they have a common neighbor in $G$ which is a locally connected vertex of $G$.

Let $G^{\prime}=G+x y$ and let $u$ be a locally connected common neighbor of $x$ and $y$. Then there is an $(x, y)$-path $P$ in $G[N(u)]$ such that $|E(P)| \geq 2$ and $E(P) \subseteq E(G[N(u)])$. The following claim is immediate.

Claim 1. The internal vertices of $P$ have degree at least 3 in $G$.

By Lemma 9 and since $h(G) \geq 2$, we have $h(\operatorname{cl}(G)) \geq 2$. Thus, by the definition of $\operatorname{cl}(G)$ and by Theorem $1, h\left(G^{\prime}\right) \geq h(\operatorname{cl}(G)) \geq 2$. By Theorem B, $E U_{h\left(G^{\prime}\right)}\left(G^{\prime}\right) \neq \emptyset$. Taking an $H \in E U_{h\left(G^{\prime}\right)}\left(G^{\prime}\right)$, we construct a subgraph $H^{\prime}$ of $G$ as follows:

$$
\begin{gathered}
V\left(H^{\prime}\right)=V(H) \backslash\left\{v \in\{x, y\}: d_{G}(v)=2 \text { and } d_{G^{\prime}}(v)=3 \text { and } d_{H}(v)=0\right\}, \\
E\left(H^{\prime}\right)= \begin{cases}E(H) & \text { if } x y \notin E(H), \\
(E(H) \Delta E(P)) \backslash\{x y\} & \text { if } x y \in E(H),\end{cases}
\end{gathered}
$$

where $E(H) \Delta(E(P)$ denotes the symmetric difference $(E(H) \backslash E(P)) \cup(E(P) \backslash E(H))$.

We show that $H^{\prime} \in E U_{h\left(G^{\prime}\right)}(G)$, i.e., $H^{\prime}$ satisfies the conditions of the definition of $E U_{h\left(G^{\prime}\right)}(G)$ for the graph $G$ and $k=h\left(G^{\prime}\right)$. Obviously, $H^{\prime}$ satisfies conditions (I) and (II). By the definition of $G+x y$ and Claim 1, all branches of length at least 2 in $G$ are the same as in $G^{\prime}$ except the case when $x$ or $y$ (or both) have degree 2 in $G$; in this exceptional case, each of $x, y$ is on a branch in $B(G) \backslash B_{1}(G)$ with adjacent endvertices and length exactly 2. Hence by Claim 1 and Lemma $6, H^{\prime}$ satisfies the other conditions of the definition of $E U_{h\left(G^{\prime}\right)}(G)$, implying $H^{\prime} \in E U_{h\left(G^{\prime}\right)}(G)$. By Theorem B, $h(G) \leq h\left(G^{\prime}\right)$, which proves Theorem 10 .

Remark 11. It was shown in [11] that the operation of contraction of an $A_{H}(F)$ contractible subgraph of a graph $H$ can be equivalently reformulated as a closure operation performed on its line graph $G=L(H)$. Combined with the closure concept for claw-free 
graphs this yields a powerful closure operation on claw-free graphs, called the $\mathcal{C}$-closure (for details we refer the reader to [11]). Theorems 7 and 10 then immediately imply that the hamiltonian index of a claw-free graph is also stable under the $\mathcal{C}$-closure operation.

Acknowledgement. The research was started when the first and second author visited the Faculty of Mathematical Sciences, University of Twente, and finished when the first author visited the Pilsner branch of the Institute of Theoretical Computer Science at the University of West Bohemia. Both authors are grateful for the hospitality extended during the visits.

\section{References}

[1] Bondy, J.A.; Murty, U.S.R.: Graph theory with applications. Macmillan, London and Elsevier, New York, 1976.

[2] Brandt, S.; Favaron, O.; Ryjáček, Z.: Closure and stable hamiltonian properties in claw-free graphs. J. Graph Theory 34 (2000), 30-41.

[3] Broersma, H.J.; Ryjáček, Z.; Schiermeyer, I.: Closure concepts - a survey. Graphs and Combinatorics 16 (2000), 17-48.

[4] Catlin, P.A.: A reduction technique to find spanning eulerian subgraphs. J. Graph Theory 12 (1988), 29-44.

[5] Harary F.; Nash-Williams C. St.J.A. : On eulerian and hamiltonian graphs and line graphs. Canad. Math. Bull. 8 (1965), 701-709.

[6] Ishizuka, S.: Closure, path-factors and path coverings in claw-free graphs. Ars Combinatoria 50 (1998), 115-128,

[7] McKee, T.A.: Recharacterizing eulerian: intimations of new duality. Discrete Math. 51 (1984), 237-242.

[8] Plummer, M.D.; Saito, A.: Closure and factor-critical graphs. Discrete Math. 215 (2000), 171-179.

[9] Ryjáček, Z.: On a closure concept in claw-free graphs. J. Combin. Theory Ser. B 70 (1997), 217-224.

[10] Ryjáček, Z.; Saito, A.; Schelp, R.H.: Closure, 2-factors and cycle coverings in clawfree graphs. J. Graph Theory 32 (1999), 109-117.

[11] Ryjáček, Z.; Schelp, R.H.: Contractibility techniques as a closure concept. Preprint 2001 (submitted).

[12] Xiong, L.: Circuits in graphs and the hamiltonian index. Ph.D Thesis, University of Twente, Enschede, The Netherlands, 2001, ISBN 9036516196.

[13] Xiong, L.; Liu, Z.: Hamiltonian iterated line graphs. Discrete Math. (to appear).

[14] Xiong, L.; Broersma, H.J.; Hoede, C; Li, X.: The hamiltonian index of a graph and its branch-bonds. Preprint 1999 (submitted). 